\title{
Traduire
}

Revue française de la traduction

$231 \mid 2014$

À table !

\section{La crème de la crème}

\section{Miranda Joubioux}

\section{(2) OpenEdition \\ Journals}

Édition électronique

URL : http://journals.openedition.org/traduire/655

DOI : 10.4000/traduire.655

ISSN : 2272-9992

\section{Éditeur}

Société française des traducteurs

\section{Édition imprimée}

Date de publication : 1 décembre 2014

Pagination : 15-19

ISSN : 0395-773X

\section{Référence électronique}

Miranda Joubioux, "La crème de la crème », Traduire [En ligne], 231 | 2014, mis en ligne le 01

décembre 2016, consulté le 01 mai 2019. URL : http://journals.openedition.org/traduire/655; DOI :

10.4000/traduire.655 


\section{La crème de la crème}

\section{Miranda Joubioux}

En Irlande, les paysages sont d'une verdure époustouflante, le climat est tempéré et, avec plus d'un million de vaches laitières, le pays est l'un des premiers producteurs laitiers du monde. C'est là que j'ai grandi. À l'époque de ma mère, on barattait le beurre manuellement à la ferme, à partir de la crème du lait de la ferme. Elle nous a transmis son amour pour le beurre et surtout pour la crème. C'est pour cela que je me suis particulièrement intéressée à la nature de la crème en Irlande, au Royaume-Uni où j'ai vécu un certain temps, et en France.

La production de la crème varie d'une crème à l'autre, et d'un pays à l'autre.

En République d'Irlande, on trouve de la fresh cream(1) et de la whipping cream(2). On ne se complique pas la vie. Depuis quelque temps, on voit également de la crème fraîche à la française. En Irlande du Nord, sous l'influence des pratiques anglaises, on trouve de la single cream, de la double cream, de la whipping cream, de la sterilised half cream et de la half cream. En France, par contre, les principales crèmes sont la crème fraîche, la crème épaisse, la crème liquide et la crème fleurette.

Avant d'expliquer les différences entre toutes ces variantes, il faut d'abord comprendre comment on fabrique la crème. II s'agit de séparer la matière grasse du lait. Ceux qui ont connu le lait livré à domicile par le laitier (en Irlande et Angleterre) sauront qu'on y trouvait souvent une séparation naturelle, la couche de crème remontant vers le haut. On pouvait donc la décanter et l'utiliser comme une single cream.

De manière industrielle, la crème se fait au moyen d'une centrifugeuse. La machine est réglée pour produire la quantité de matière grasse désirée. Ensuite, la crème est pasteurisée pour tuer les bactéries. Plus elle est pasteurisée, plus son goût et sa durée de vie sont altérés.

(1) II ne s'agit pas de crème fraîche, mais de crème liquide sans ajout de ferments lactiques.

(2) Crème à fouetter. 
Cependant, il reste encore des méthodes plus traditionnelles, telles que la méthode de la clotted cream ou de la Devonshire cream. Le lait de vache est d'abord filtré, puis laissé dans un grand récipient peu profond jusqu'à ce que la crème remonte. L'ensemble est chauffé au bain-marie à $80-90^{\circ} \mathrm{C}$ pendant $40-45$ minutes. Au bout de 24 heures, une couche de crème se forme avec une croûte solide que l'on peut facilement séparer du résidu de lait au-dessous. Cette méthode crée une crème à plus de $55 \%$ de matière grasse. Elle est encore utilisée dans les îles Anglo-Normandes et dans le Devon, le Somerset et les Cornouailles. Désormais, il existe des méthodes plus industrielles pour faire de la clotted cream. Mais quelle en est l'utilisation? On la mange souvent en accompagnement des scones, une pâtisserie écossaise.

Revenons aux différents types de crème. La double cream n'est pas une crème épaisse. C'est une crème liquide contenant au moins $48 \%$ de matière grasse. Cette crème peut être fouettée, mais difficilement. Cela demande plus d'effort qu'avec une whipping cream. On la verse souvent sur les desserts tels que les tartes aux pommes (apple pie). Le goût n'est pas du tout acide, puisqu'il n'y a pas de fermentation (souring). En France, les crèmes liquides sont souvent légèrement maturées par l'ajout de ferments. C'est pour cela qu'une crème liquide française ne s'utilise pas de la même façon.

La single cream est une crème liquide. Elle contient au moins $18 \%$ de matière grasse et elle n'est pas stérilisée. On l'utilise dans le café, on la verse sur les desserts et on l'intègre dans les soupes et autres plats salés.

Qu'est-ce que la whipping cream, alors ? C'est une crème avec au moins $35 \%$ de matière grasse et un fort pouvoir de foisonnement, ce qui permet d'y incorporer de l'air pour la rendre plus légère et volumineuse. Elle pourrait se comparer à la crème fleurette en France, qui contient $30 \%$ de matière grasse.

La sterilised cream contient au moins $12 \%$ de matière grasse et a été stérilisée, ce qui tue quasiment toutes les bactéries et permet une conservation de plusieurs mois hors réfrigérateur, avant ouverture. On l'appelle aussi UHT cream (ultra-high temperature), car la stérilisation se fait à très haute température.

La half cream est une crème à $12 \%$ de matière grasse, mais qui n'a pas été stérilisée.

II fut un temps où l'on trouvait encore de la sour cream en Irlande et au Royaume-Uni, une sorte de crème fraîche. Elle contient au moins $18 \%$ de matière grasse et on lui ajoute des ferments lactiques qui en modifient le goût, la rendant plus acidulée et plus épaisse.

Désormais, on trouve très souvent de la " crème fraîche " (à la française) dans les îles britanniques. C'est très à la mode ; cet engouement s'explique par les nombreuses émissions de télévision relatives à la cuisine. Le pourcentage de matière grasse est précisé sur les emballages avec des versions " light ", comme en France. Elle est un peu moins acidulée que la sour cream. 
Pour ce qui est des crèmes françaises, la crème entière contient au moins $30 \%$ de matière grasse. Le fait qu'elle soit liquide ou épaisse n'influence que sa texture, son goût, plus ou moins acidulé, et son comportement en cuisine. Sa texture n'a rien à voir avec le taux de matière grasse. On la reconnaît souvent par son capuchon rouge pour ce qui est de la version liquide.

La crème légère (bouchon bleu) compte entre 12 et $30 \%$ de matière grasse.

Ces deux crèmes sont des crèmes fraîches. Elles ont été pasteurisées et ensemencées avec des ferments lactiques, ce qui leur donne leur goût et leur consistance.

Quelle que soit sa forme, la crème en France est maturée avec des ferments lactiques, à l'exception de la crème fleurette, qui est simplement pasteurisée (sauf mention contraire).

La crème anglaise, dont la traduction anglaise est souvent custard, n'est pas de la famille des crèmes ; il s'agit d'une sauce sucrée faite avec des œufs et du lait.

La Chantilly est une crème fouettée (souvent UHT) aromatisée à la vanille et avec du sucre ajouté.

La crème a toute son importance dans la cuisine anglaise. Quand on entre dans une pâtisserie en Irlande ou au Royaume-Uni, on voit des quantités de pâtisseries à la crème (fouettée, avec ou sans sucre ajouté). Quand on parle de pâtisseries à la crème en France, il s'agit le plus souvent de crème pâtissière, préparée avec du lait, du sucre, de la farine et des œufs, ou de crème au beurre, fabriquée avec du beurre et des œufs, et donc très riche.

Naturellement, la langue anglaise a sa part d'expressions idiomatiques faisant référence à la crème :

The cream of the crop

Le meilleur de tous, impressionnant, le meilleur choix, le fleuron

The cream of society

Le beau monde, l'élite, la jet set, la bourgeoisie

The cat that got the cream

Une personne très satisfaite d'elle-même ou suffisante

There are more ways of choking a cat than killing it with cream

$\|$ y a plusieurs façons d'atteindre son objectif

To cream someone

Vaincre ou détruire quelqu'un

Cream rises to the top

La qualité d'une personne ou d'une idée finit toujours par se faire remarquer 
Peaches and cream

Quelque chose de très plaisant

Qualifie aussi un teint doux sans imperfection

He's a cream puff (argot)

Quelqu'un de faible, une mauviette, une poule mouillée

Crème de la crème

Les meilleurs, la crème (même signification que Cream of the crop)

\section{Comment trouver une traduction convenable ?}

Nous, traducteurs, savons qu'une recette ou qu'une ligne sur la carte d'un restaurant peut être difficile à traduire, parce qu'il n'y a pas forcement d'équivalent en français ou en anglais. Pour ce qui est des recettes, voici quelques conseils : si vous traduisez de l'anglais en français, faites attention à votre choix de vocabulaire concernant la crème. S'il s'agit d'une recette salée et que la crème est chauffée, alors il suffit de choisir l'équivalent en pourcentage de matière grasse. Le petit goût acidulé que l'on trouve dans les crèmes françaises ne changera pas trop le résultat.

Un exemple qui démontre que chaque cas se traite individuellement : les tagliatelles carbonara. Selon les recettes, ce plat est réalisé avec de la single cream (Jamie Oliver), de la double cream (BBC Good Food \& Deliaonline.com), de la thickened cream (Taste.au) ou de la heavy cream (www.foodandwine.com USA). Pour les deux derniers exemples, on optera dans la traduction pour l'expression " crème fraîche ". On pourra traduire single cream par "crème liquide légère " et double cream par "crème liquide entière ". Sour cream pourra se traduire par "crème fraîche épaisse ". Et les mêmes questions se poseront pour les soupes, les quiches, etc.

Si le plat est sucré, les choses se compliquent. De manière générale, la crème qui est incorporée dans une recette anglaise sucrée n'a pas le goût acidulé si familier en France. Pour certaines recettes chauffées ou acidulées par nature, comme le cheesecake ou la custard tart (flan pâtissier), cela n'a pas vraiment d'importance. Par contre, pour la plupart des recettes sucrées, il faudra utiliser une crème sans ferments. La whipping cream peut être traduite par " crème fleurette ", qui n'a pas de ferments lactiques. II faut savoir qu'on peut également utiliser la crème fleurette sans la battre pour faire office de double cream. Par exemple, on pourra traduire la double cream utilisée pour faire une glace (ice-cream) maison par " crème fleurette liquide ". Cela évitera une glace au goût acidulé.

Quand on traduit la crème qui accompagne une recette ou un plat, on reste dans le même esprit. Face à des spécialités telles que la clotted cream qui accompagne les scones, il faut laisser le terme tel quel, quitte à l'expliquer avec une note de bas de page ou entre parenthèses. 
Dans le sens contraire, du français en anglais, on a plus de liberté. C'est la matière grasse qui importe.

Si vous aimez cuisiner ou même préparer votre Irish Coffee pour agrémenter les longues soirées d'hiver, vous le saurez : la crème est tout un art !

miranda.joubioux@art4u.fr

Miranda Joubioux est de nationalité irlandaise. Elle a obtenu une maîtrise de linguistique à l'University College Dublin en 1987. Traductrice du français vers l'anglais depuis 1996, elle exerce en indépendante depuis 12 ans. Ses spécialisations sont le nautisme, la marine, l'architecture, la construction et le marketing. Pour plus d'informations : www.art4u.fr 